\title{
Objective Physical Activity Assessment in Clinical Congenital Heart Disease Research: A Systematic Review on Study Quality, Methodology, and Outcomes
}

\author{
Leon Brudy $^{a}$ b Michael Meyer ${ }^{a} \quad$ Luisa Garcia-Cuenllas ${ }^{c}$ Renate Oberhoffer ${ }^{a, b}$ \\ Alfred Hager ${ }^{\mathrm{a}}$ Peter Ewert ${ }^{\mathrm{a}} \quad$ Jan Müller ${ }^{\mathrm{a}, \mathrm{b}}$ \\ aDepartment of Pediatric Cardiology and Congenital Heart Disease, Deutsches Herzzentrum München, Technische \\ Universität München, Munich, Germany; 'bnstitute of Preventive Pediatrics, Technische Universität München, \\ Munich, Germany; ${ }^{C}$ Department of Pediatric Cardiology, Hospital Sant Joan de Dèu, Barcelona, Spain
}

\section{Keywords}

Accelerometers - Congenital heart disease - Physical activity . Prevention exercise

\begin{abstract}
Background: The shift toward a preventative approach in medical aftercare of congenital heart disease (CHD) patients has led to encouragement of regular physical activity (PA) in this patient population. Objective measures are crucial in accurately displaying PA levels and have increasingly found their way into clinical research. This review aims to give an overview about quality, methodology, and outcomes of current scientific work on accelerometers objectively assessing PA in patients with CHD. Methods: Systematically researched literature in all relevant databases (PubMed, Cochrane, and Scopus) over the past decade (2009-2019) with history of CHD and accelerometer-based PA assessment was evaluated by 2 independent reviewers according to the Study Quality Assessment Tool for Observational Cohort and Cross-Sectional Studies of the National Heart, Lung, and Blood Institute. Results: Eight articles with 664 pediatric patients with CHD aged 3-18 years (range 10-162 patients), 5 studies with 574 adults with CHD aged 18-63 years (range 28-330 pa-
\end{abstract}

tients), and 3 studies with 177 pediatric patients and adults with $\mathrm{CHD}$ aged 8-52 years were included. Two studies were rated "good"; 9, "fair"; and 5, "poor." Methodologies and devices differed substantially across all studies. Conclusions: Overall study quality was fair at best, and due to difficult methodological comparability of the studies, no clear answer on how active patients with CHD really are can currently be given. Larger studies carefully considering collection and processing criteria, and correct reporting standards exploring PA in patients with CHD from different angles are needed.

(c) 2021 S. Karger AG, Basel

\section{Introduction}

Regular physical activity (PA) has long been known as one of the most effective preventative tools for a vast number of diseases and clinical conditions, especially those of cardiovascular origin $[1,2]$. Patients with congenital heart disease (CHD) have inhibition to different extends within their cardiovascular system since birth, and with increased survival, medical aftercare has shifted toward prevention of secondary diseases in this patient

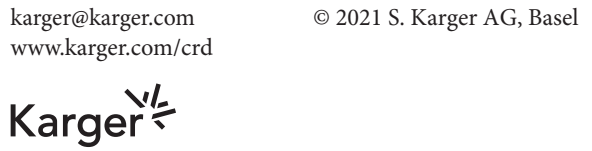

Leon Brudy

Institute of Preventive Pediatrics, Technische Universität München Georg-Brauchle-Ring 60/62 DE-80636 Munich (Germany)

leon.brudy@tum.de 
Fig. 1. Search and selection process for systematic review according to Preferred Reporting Items for Systematic Reviews and Meta-Analyses (PRISMA).

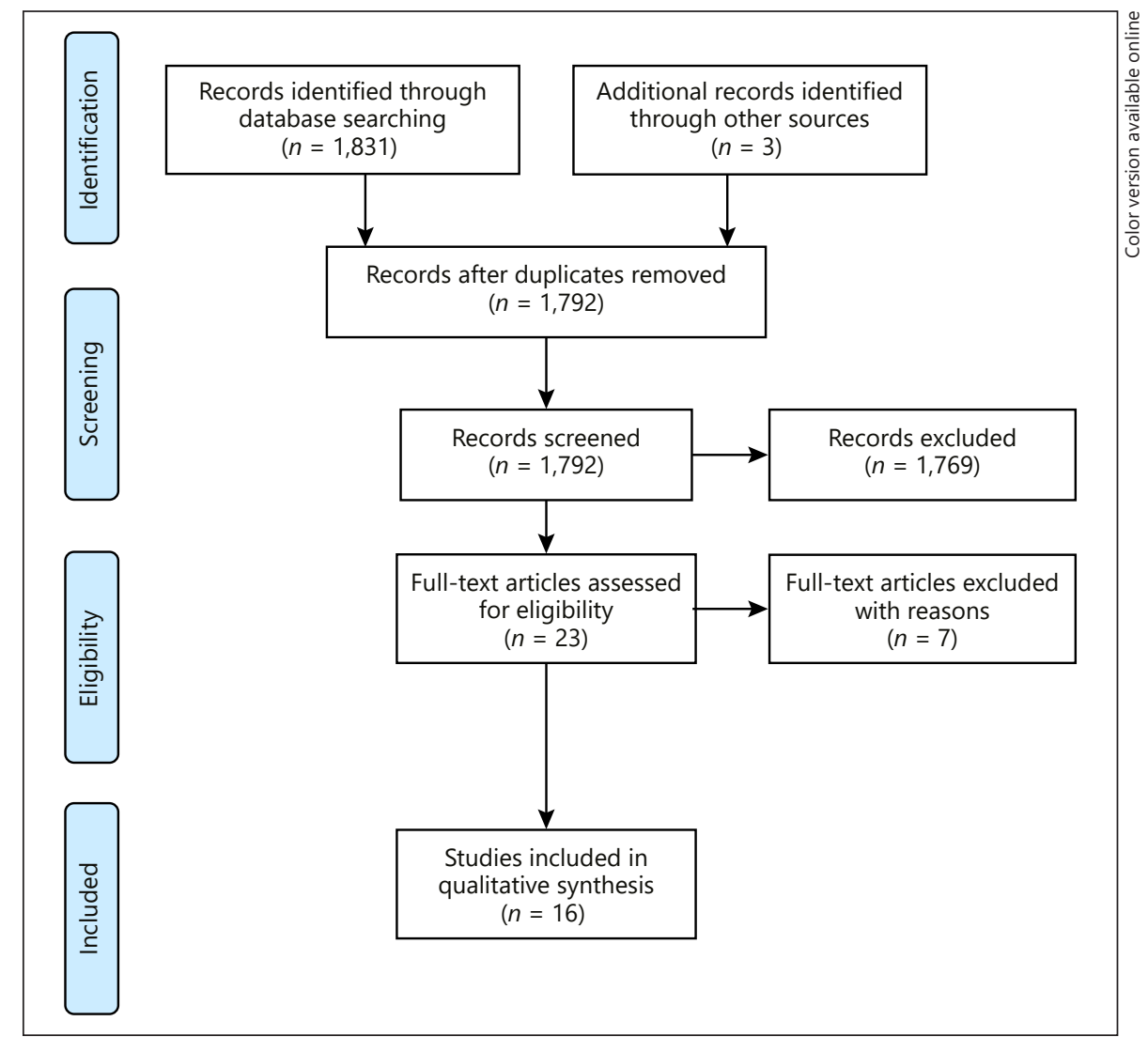

population [3]. Part of this preventative approach is the encouragement to regular PA and sports participation [4] as it is now considered beneficial without additional risk for almost all patients with CHD. The encouragement toward a generally physically active lifestyle becomes imperative in this context [3]. Technological advances offer exciting research opportunities [5] and since subjectively estimated PA fails to draw accurate pictures [6], objective measures to assess PA have also found their way into clinical CHD research. After more than a decade of research assessing PA with accelerometers in CHD patients, it is time to evaluate where the field is at and where it needs to go from there. Therefore, this review aimed to give an overview about quality, methodology, and outcomes of current scientific work on accelerometers objectively assessing PA in CHD patients.

\section{Material and Methods}

\section{Search Strategy}

A systematic literature research referring to January 2009 until December 2019 was conducted in the electronic databases PubMed, Cochrane, and Scopus. Two independent reviewers identified rel- evant original articles and randomized controlled trials (RCT) in English language. A standard protocol with search terms was generated according to the population, intervention, comparison, outcome, context (PICO-C) [7] method and connected as follows:

1. Congenital heart disease OR congenital heart diseases OR congenital heart defect OR congenital heart defects.

2. Physical activity assessment OR physical activity measurement OR physical activity OR daily activity OR accelerometer OR accelerometry OR wearable OR activity tracker OR activity monitor OR fitness tracker.

Medical Subject Headings terms and similar filters (clinical trial, randomized controlled trial, published in the last 10 years, humans, English language) were used and appropriately adapted if necessary. According to the latest PRISMA statement [8], supplementary reference lists were analyzed to detect further eligible articles.

\section{Data Collection}

Both reviewers screened all relevant articles for titles and abstracts which had to fulfill the basic inclusion criteria: history of CHD and accelerometer-based PA assessment. PA had to be classified in some sort of PA intensity and not merely step count alone as moderate-to-vigorous physical activity (MVPA) is shown to be beneficial for cardiovascular health [9].

At least one of the two reviewers had to consider a reference eligible. A third reviewer was consulted to resolve disagreement before and during full-text analysis. 
Table 1. Quality assessment according to the NHLBI Quality Assessment Tool for Observational Cohort and Cross-Sectional Studies

\begin{tabular}{llllllllllllllllllll}
\hline Study & Type Q1 & Q2 & Q3 & Q4 & Q5 & Q6 & Q7 & Q8 & Q9 & Q10 & Q11 Q12 & Q13 & Q14 & Quality \\
rating
\end{tabular}

Pediatric CHD $(n=8)$

Kao et al. [18]

Ewalt et al. [19]

Longmuir et al. [20]

Stone et al. [21]

Klausen et al. [22]

Banks et al. [23]

Voss et al. [24]

Brudy et al. [25]

$\begin{array}{llllll}\operatorname{CSS} & \sqrt{ } & \sqrt{ } & \sqrt{ } & - & - \\ \operatorname{CSS} & \sqrt{ } & \sqrt{ } & \sqrt{ } & \sqrt{ } & \sqrt{ } \\ \operatorname{RCT} & \sqrt{ } & \sqrt{ } & \sqrt{ } & \sqrt{ } & \sqrt{ } \\ \operatorname{CSS} & \sqrt{ } & \sqrt{ } & \sqrt{ } & - & - \\ \operatorname{RCT} & \sqrt{ } & \sqrt{ } & \sqrt{ } & \sqrt{ } & \sqrt{ } \\ \operatorname{CSS} & \sqrt{ } & \sqrt{ } & - & - & - \\ \operatorname{CSS} & \sqrt{ } & - & \operatorname{CD} & \sqrt{ } & - \\ \operatorname{CSS} & \sqrt{ } & \sqrt{ } & - & \sqrt{ } & -\end{array}$

$\begin{array}{lllll}\sqrt{ } & - & - & \sqrt{ } & \text { NA } \\ - & - & \text { NA } & \sqrt{ } & - \\ \sqrt{ } & \sqrt{ } & - & \sqrt{ } & \sqrt{ } \\ - & - & \text { NA } & - & - \\ \sqrt{ } & & - & \sqrt{ } & \sqrt{ } \\ \sqrt{ } & \sqrt{ } & \sqrt{ } & \sqrt{ } & - \\ \sqrt{ } & - & \text { NA } & \sqrt{ } & - \\ \sqrt{ } & \sqrt{ } & \sqrt{ } & \sqrt{ } & -\end{array}$

$\begin{array}{ll}\sqrt{ } & - \\ \sqrt{ } & - \\ \sqrt{ } & \sqrt{ } \\ \sqrt{ } & - \\ \sqrt{ } & \sqrt{ } \\ \sqrt{ } & - \\ \sqrt{ } & - \\ \sqrt{ } & -\end{array}$

$\begin{array}{llll}- & \sqrt{ } & - & \text { Poor } \\ - & \sqrt{ } & - & \text { Fair } \\ \sqrt{ } & \sqrt{ } & \sqrt{ } & \text { Good } \\ - & \sqrt{ } & - & \text { Poor } \\ \sqrt{ } & \sqrt{ } & \sqrt{ } & \text { Good } \\ - & \sqrt{ } & - & \text { Poor } \\ - & \sqrt{ } & \sqrt{ } & \text { Poor } \\ - & \text { CD } & \sqrt{ } & \text { Fair }\end{array}$

ACHD $(n=5)$

Dua et al. [26]

Müller et al. [27]

Sandberg et al. [28]

Opotowsky et al. [29]

Larsson et al. [30]

$\begin{array}{lllllll}\operatorname{CCT} & \sqrt{ } & \sqrt{ } & - & \sqrt{ } & - & \sqrt{ } \\ \operatorname{CSS} & \sqrt{ } & \sqrt{ } & - & \sqrt{ } & - & - \\ \operatorname{CSS} & \sqrt{ } & \sqrt{ } & \sqrt{ } & \sqrt{ } & - & \sqrt{ } \\ \operatorname{RCT} & \sqrt{ } & \sqrt{ } & \sqrt{ } & \sqrt{ } & - & \sqrt{ } \\ \operatorname{CSS} & \sqrt{ } & \sqrt{ } & \sqrt{ } & \sqrt{ } & \sqrt{ } & \sqrt{ }\end{array}$

$\sqrt{ }$

$\begin{array}{lllllllll}\sqrt{ } & \sqrt{ } & \sqrt{ } & \sqrt{ } & - & \sqrt{ } & - & \text { Fair }\end{array}$

NA $\sqrt{ } \quad-\quad \sqrt{ } \quad-\quad \sqrt{ } \quad \sqrt{ }$ Fair

NA $\sqrt{ } \quad-\quad \sqrt{ } \quad-\quad \sqrt{ } \quad \sqrt{ }$ Fair

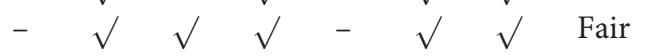

Pediatric and ACHD $(n=3)$

Müller et al. [31]

Duppen et al. [32]

Hedlund et al. [33]

$$
\begin{array}{llllllllllllllll}
\operatorname{CSS} & \sqrt{ } & \sqrt{ } & \sqrt{ } & \sqrt{ } & \sqrt{ } & - & - & - & \sqrt{ } & - & \sqrt{ } & - & \sqrt{ } & - & \text { Fair } \\
\operatorname{RCT} & \sqrt{ } & \sqrt{ } & - & - & \sqrt{ } & \sqrt{ } & \sqrt{ } & - & \sqrt{ } & \sqrt{ } & \sqrt{ } & - & \sqrt{ } & - & \text { Fair } \\
\operatorname{CCS} \sqrt{ } & - & \sqrt{ } & - & - & - & - & \text { NA } & \sqrt{ } & - & \sqrt{ } & - & \sqrt{ } & - & \text { Poor }
\end{array}
$$

CD, cannot determine; NA, not applicable; $\sqrt{ }$ denotes “yes"; - denotes "no"; RCT, randomized controlled trial; CCT, controlled clinical trial; CSS, cross-sectional study; CHD, congenital heart disease; ACHD, adults with congenital heart disease; NHLBI, National Heart, Lung, and Blood Institute.

Both reviewers rated the included literature according to the Study Quality Assessment Tool for Observational Cohort and Cross-Sectional Studies of the National Heart, Lung, and Blood Institute [10] - a 14-item list assessing potential risk for bias - and consequently categorized them as good, fair, or poor.

\section{Results}

\section{Description of Selected Studies}

Figure 1 displays the search and selection process. Reasons for exclusion after full-text analysis were: One study protocol [11], two validation studies [12,13], four studies without reporting PA intensity [14-17]. Overall, 16 articles with a total of $1,415 \mathrm{CHD}$ patients met all inclusion criteria.

\section{Study Characteristics}

Eight studies [18-25] evaluated 664 pediatric patients with CHD aged 3-18 years (range 10-162 patients), 5 stud- ies [26-30] 574 ACHD aged 18-63 years (range 28-330 patients). Three studies [31-33] with 177 patients (range 3099 patients) examined both pediatric patients and adults with CHD aged 8-52 years but did not report results separately for these age ranges. Detailed information on study types and quality rating can be found in Table 1 .

The majority of cross-sectional studies (CSS) compared their $\mathrm{CHD}$ population to a healthy reference cohort (RC) $[18,19,21,25,28,30,33]$; the remaining $[23,24,27$, 31] compared PA within their CHD sample. All but 4 studies [20, 22, 23, 29] compared their patients' PA to a healthy RC, against varying institutional PA recommendations, or against both. All CSS evaluated total daily (and where applicable weekly) PA, MVPA, or separate PA intensities including sedentary time. Subjective PA was also compared in seven CSS $[18,21,25,30,33]$; three CSS [23, 27, 31] and one RCT [32] examined QoL; and three CSS exercise capacity parameters $[23,27,31]$ in relation to PA.

Intervention studies lasted 12 -months in pediatric patients with CHD [20,22], 12 weeks in adolescents and 


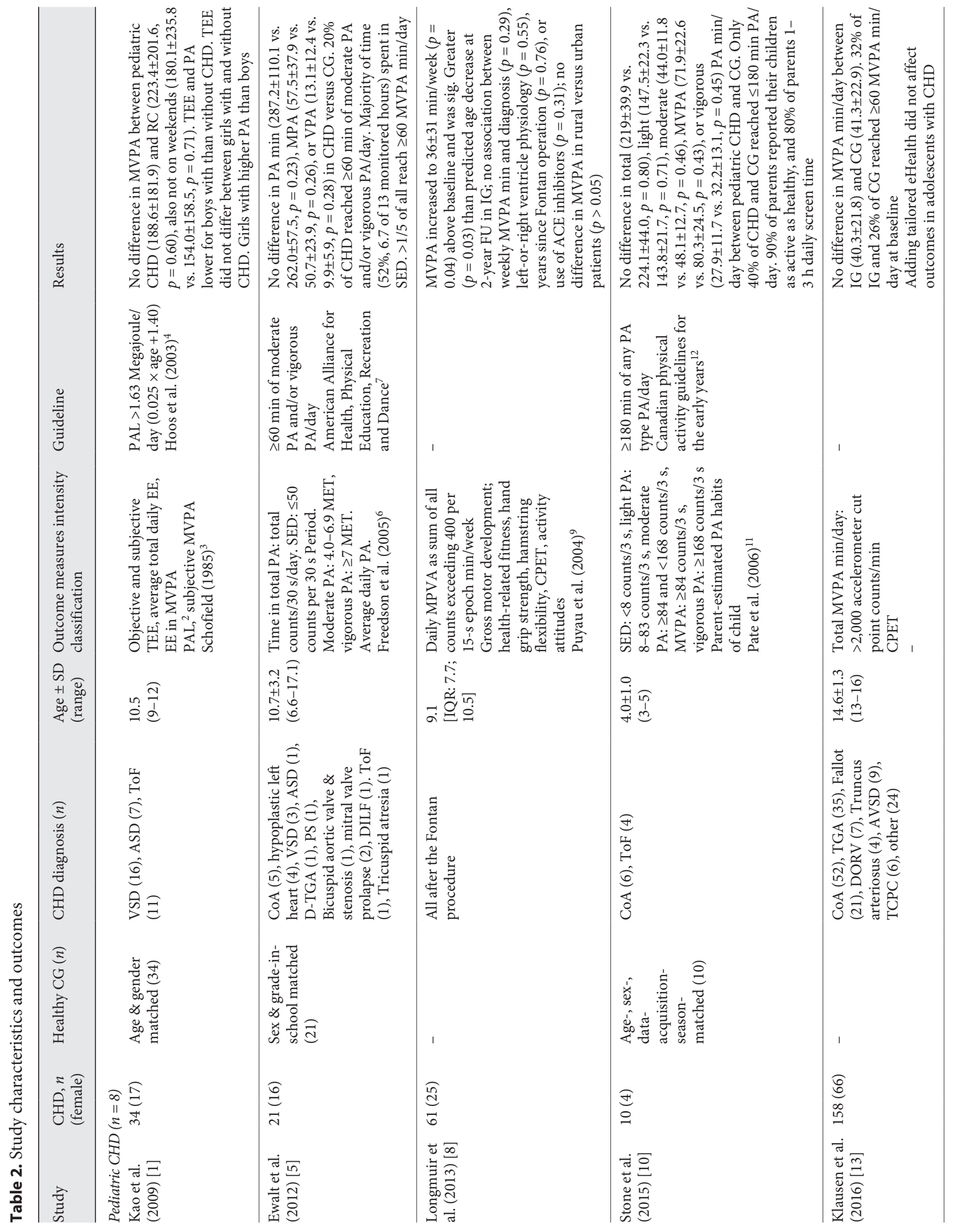




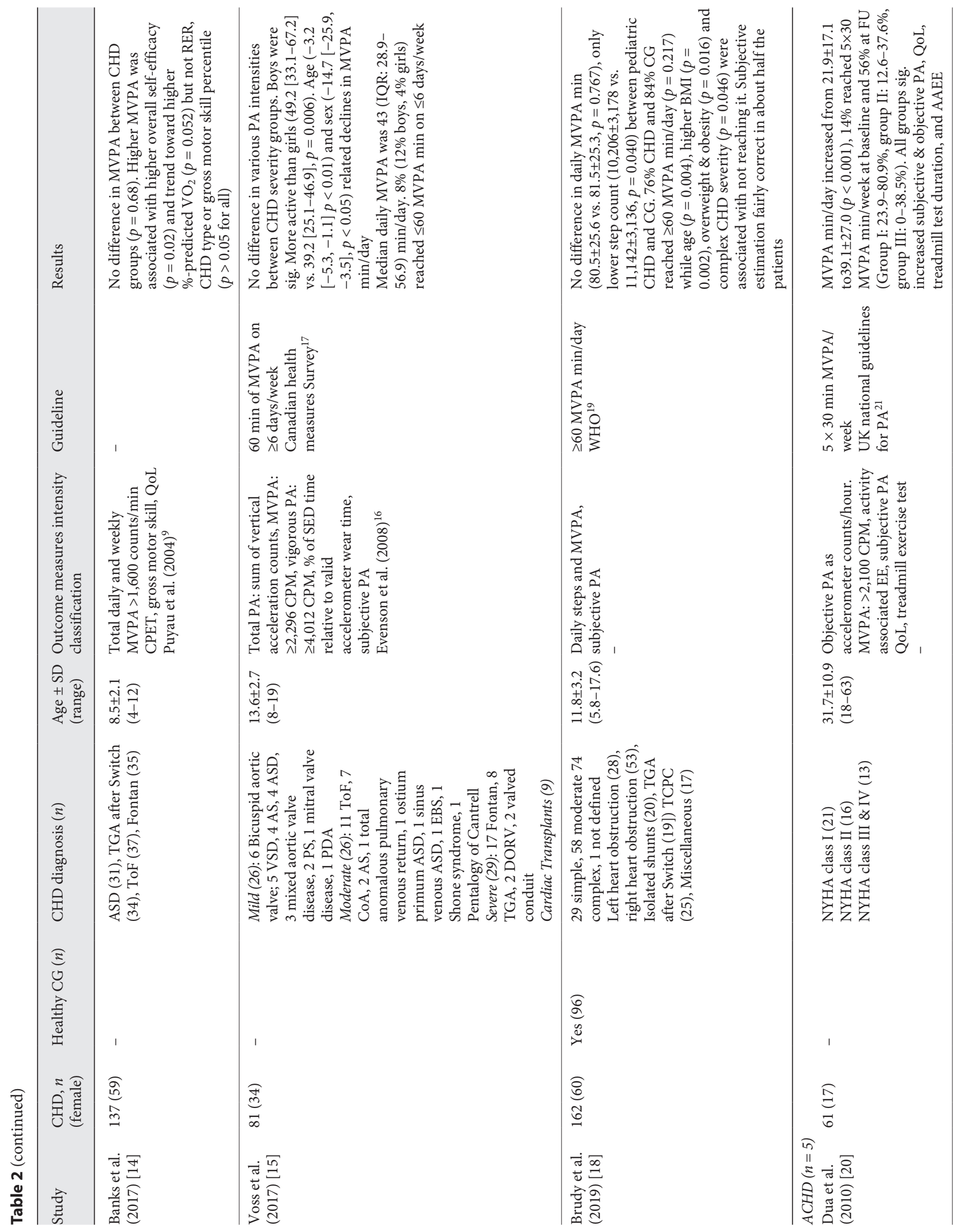




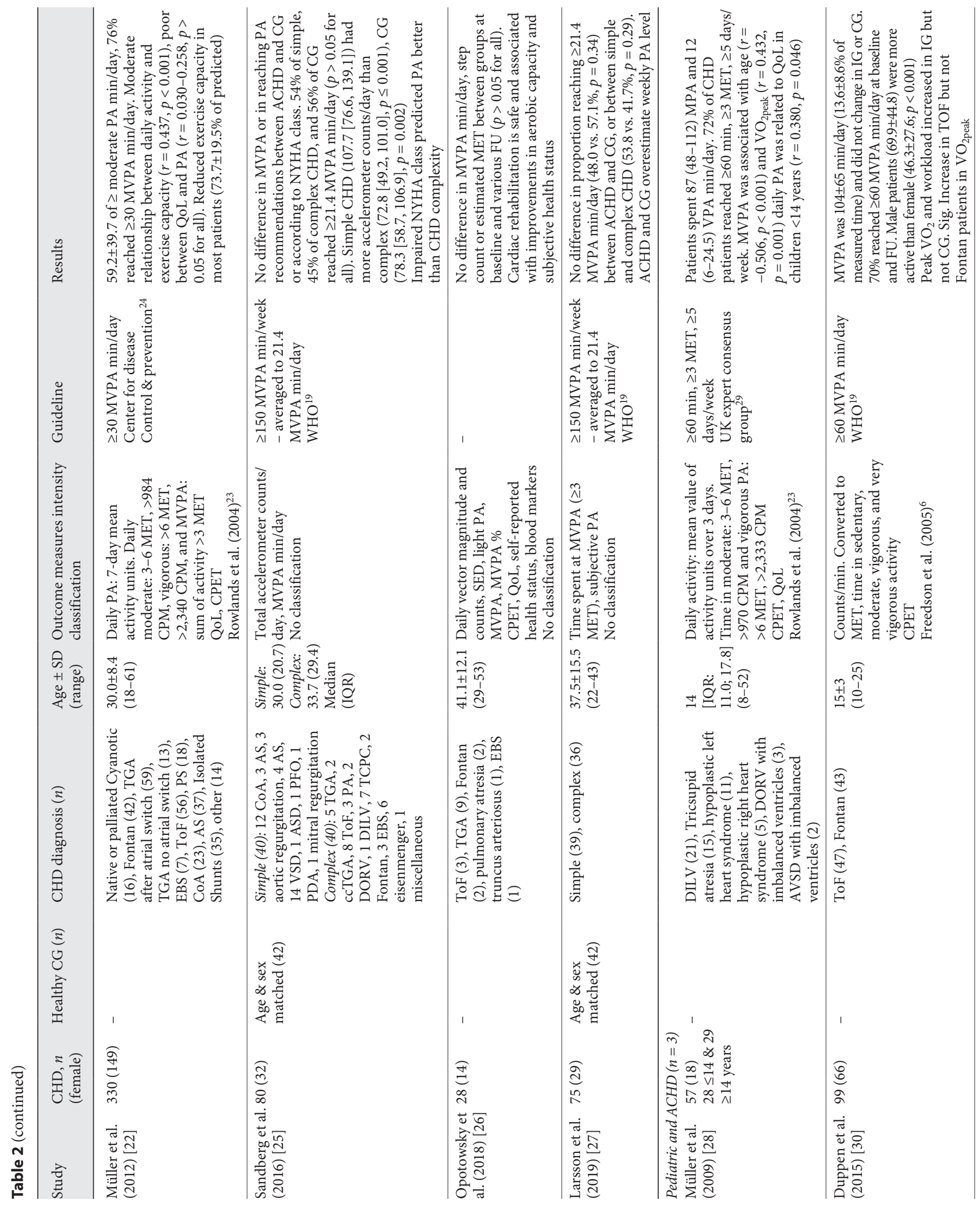




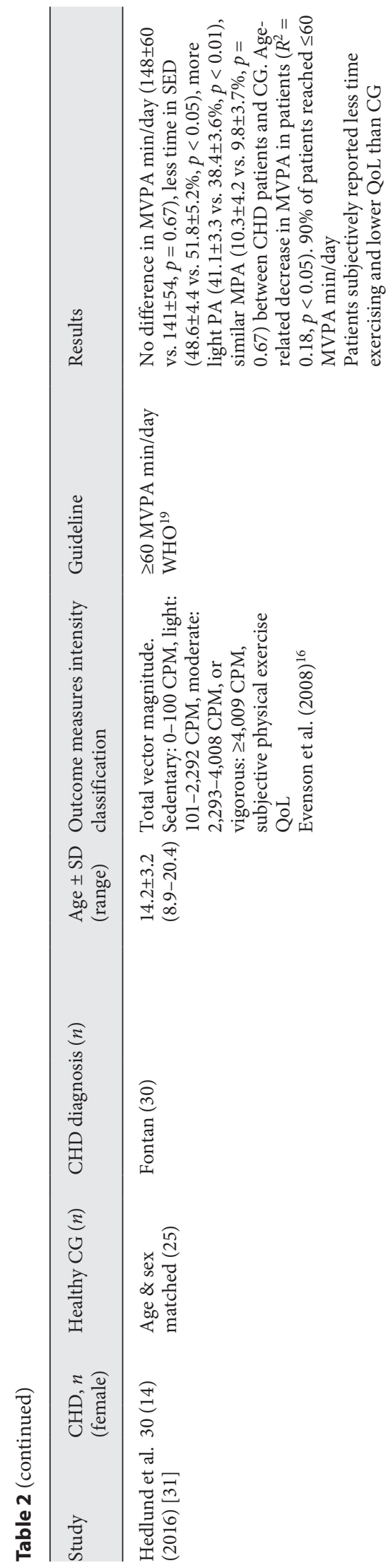

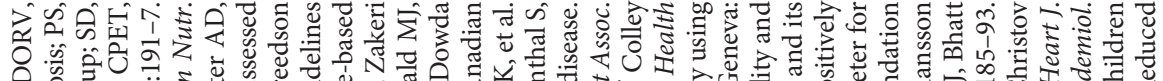

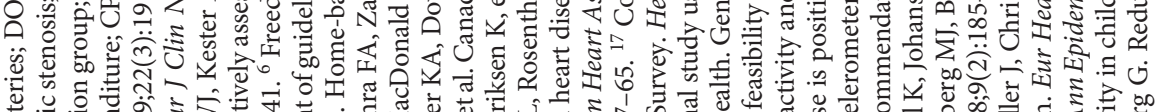

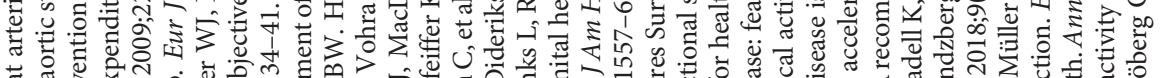

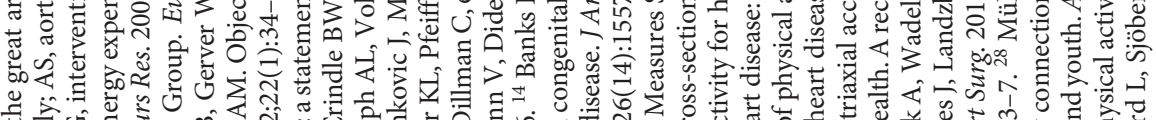

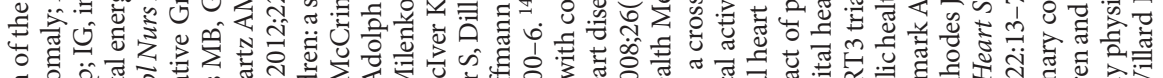

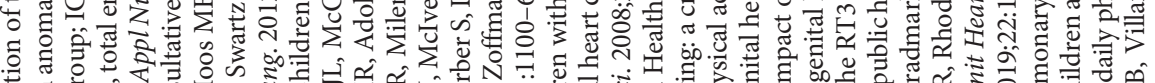

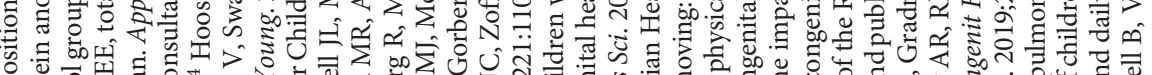

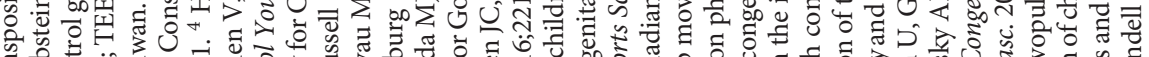

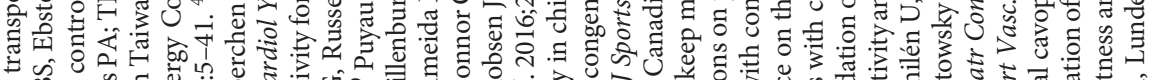

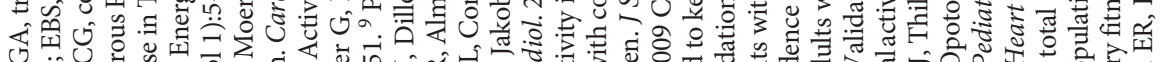

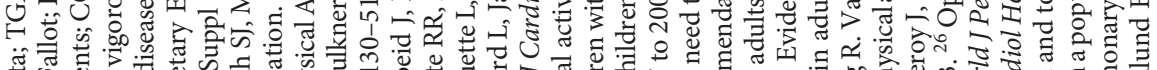

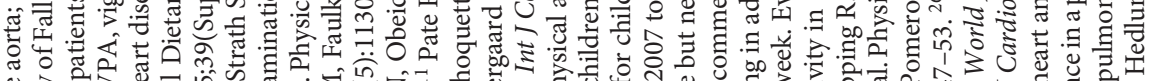

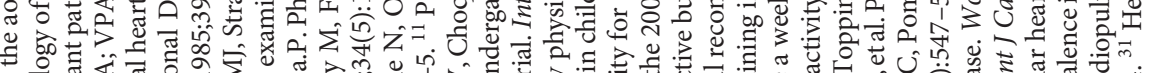

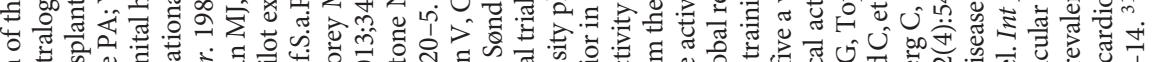
表 की

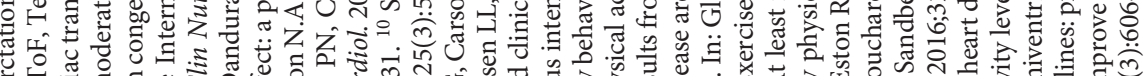

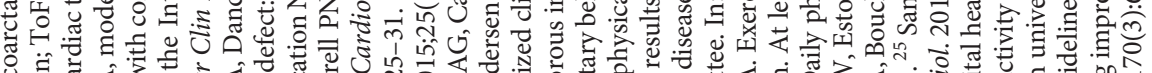
8.

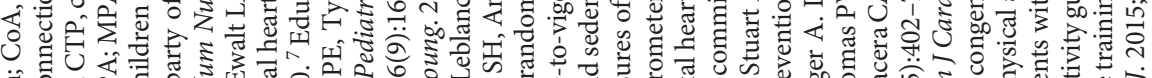

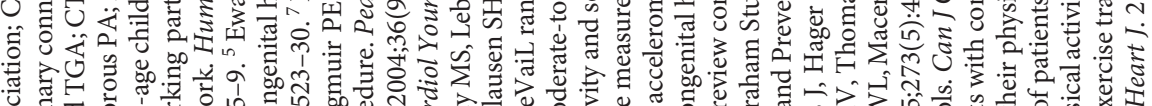

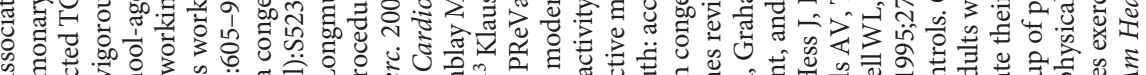

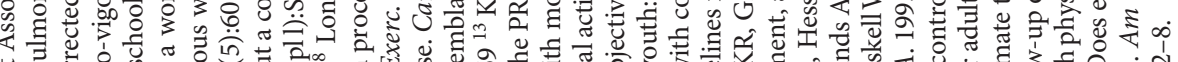

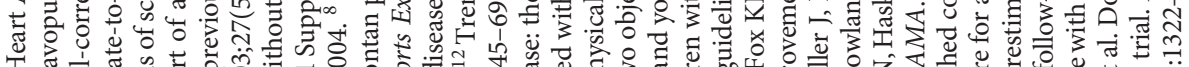

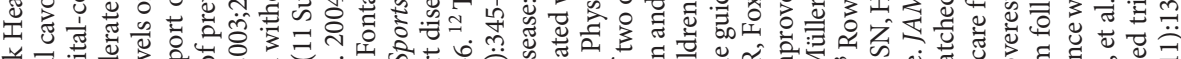

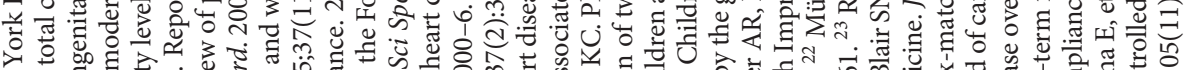

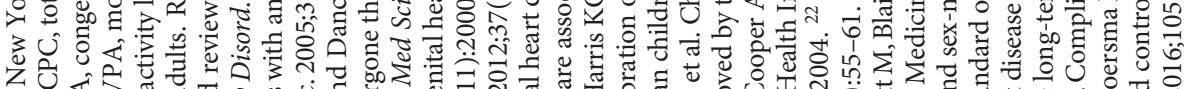

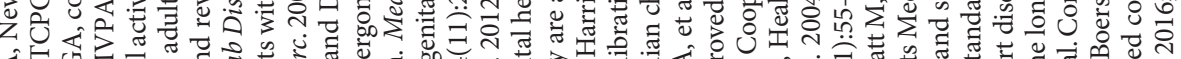

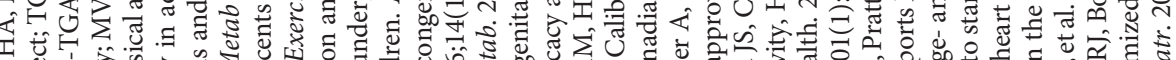

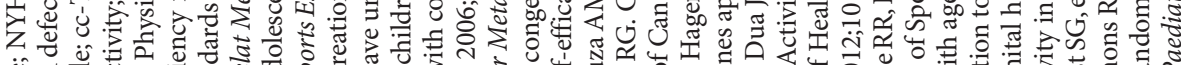

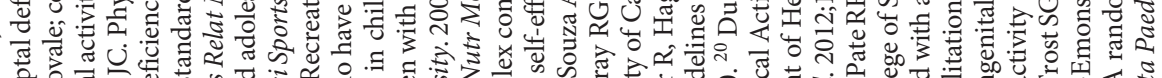

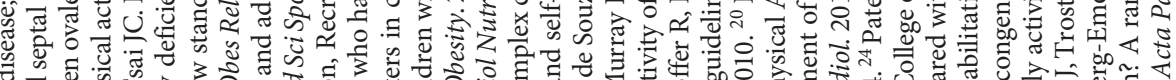

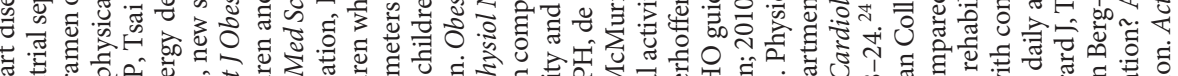

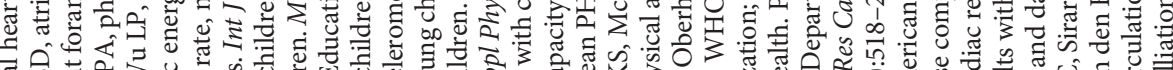

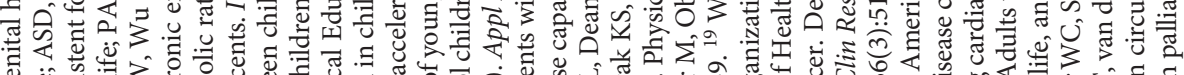

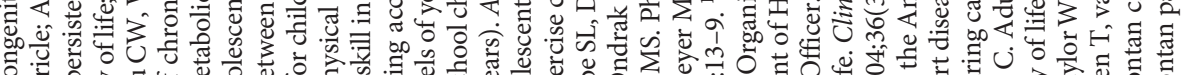

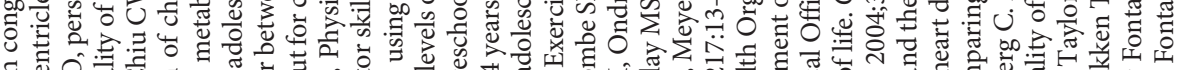

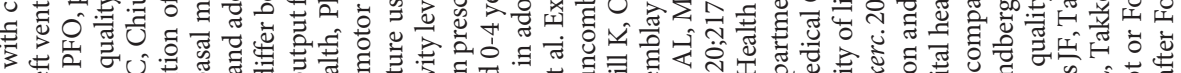

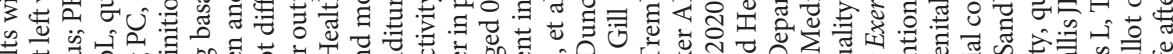

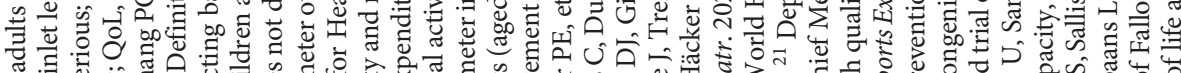

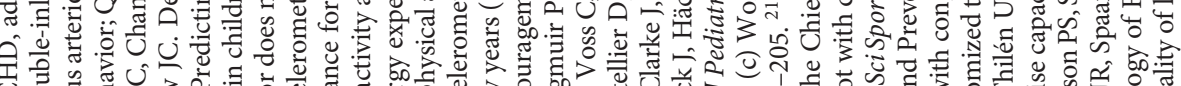

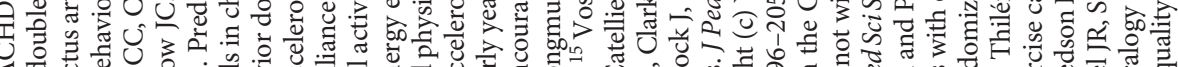

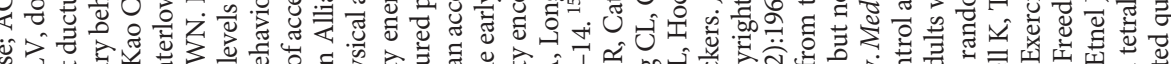

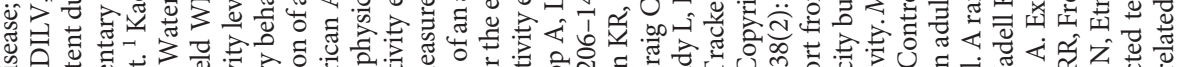

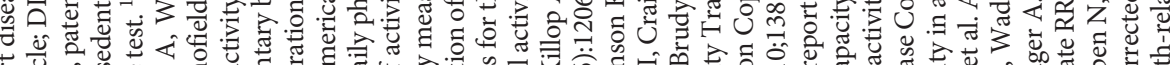

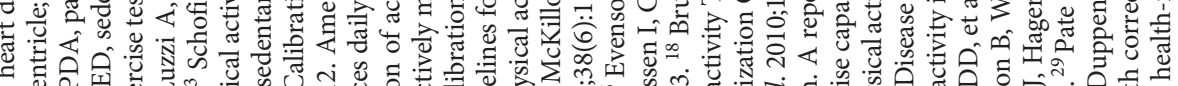

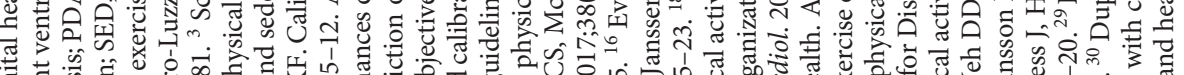

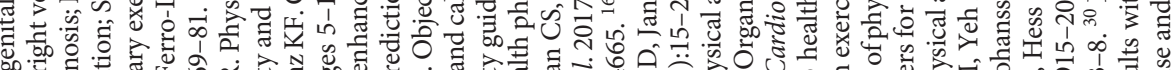

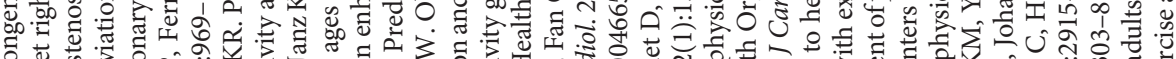

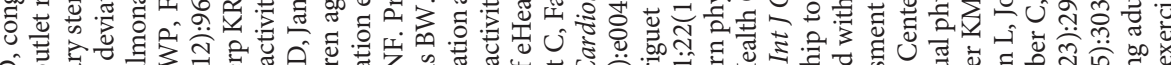
คे I

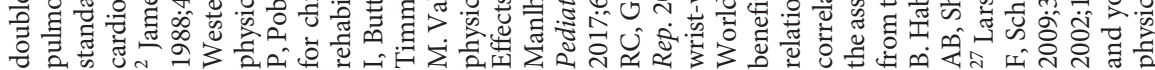


young adults with CHD [32], and 10 or 12 weeks in ACHD [26, 29]. Alongside increasing PA these studies also focused on other parameters beyond of the scope of this review (exercise capacity, QoL, and gross motor skill). Table 2 provides further information on cohort description, outcome measures, and PA classifications.

\section{Accelerometers Used}

Detailed information on technicalities and characteristics of the devices used in all included studies can be found in Table 3. The majority of studies aimed to collect data on PA for all seven days of the week [19-21, 23-27, 29, 33]; one study collected data for six [22] and five [32] days, two sampled for four days $[28,30]$, and two older studies $[18,31]$ assessed PA over three days. All studies recorded activity during waking hours. Sandberg et al. [28], Larsson et al. [30], and Hedlund et al. [33] additionally utilized a 24 -h protocol.

Sampling epoch length of the accelerometer devices differed between the studies [19-28, 30, 31, 33] and ranged from 3 to 60 seconds. Three studies $[18,29,32]$ did not report about their devices' sampling epochs at all. Only Voss and colleagues [24] and Hedlund and colleagues [33] reported the sampling frequency $(30 \mathrm{~Hz})$ of their devices. Detailed listings of frequency and epoch are displayed in Table 3.

Daily PA in different levels of activity (sedentary, light, moderate, and vigorous) or MVPA were assessed in all studies. However, thresholds, wear-time algorithm and PA intensity classification for different activity levels differed or were not reported at all. Four studies [25, 28-30] did not report how accelerometer data were transferred into PA intensity; in two studies $[22,26]$ PA could not be determined.

\section{Discussion}

\section{Study Quality}

The majority of studies were rated "fair" at best with more than a third showing substantial risk for bias with a "poor" rating. Qualitative flaws were mostly found in missing power or effect size, low participation rate or very small cohorts. The lack of key confounding variables in the analysis, no blinding of accessors and an unclear subject selection (Table 1) leaves room for stronger studies in the future. Major statistical and methodological flaws like reporting inconsistent $p$-values in the abstract versus results section [23] or calculating PA thresholds using an adult recommendation [34] for a cohort of preschool children [18] - should be avoided.

Objective Physical Activity Assessment in Clinical CHD Research

\section{Accelerometer Characteristics}

Unless sleep data are relevant, a protocol sampling during waking hours with minimum of four valid data days including one weekend day is recommended [35]. A 7 -day protocol is even more advisable, especially in younger participants [36] Three studies [28, 30, 33] utilized a 24-h recording protocol, the rest sampled just during waking hours. Only 3 separate studies $[19,20,25]$ actually adhered to these valid-day inclusion criteria (Table 3).

All but two studies [25, 33] used hip-worn devices. Overall, a possible trade-off between classification accuracy and compliance in hip- versus wrist-worn devices needs to be considered. Ideally studies should be designed with both hip- and wrist-worn devices.

Only two studies reported sampling frequency [24,33] and none data filters of their devices. Even though exact information tends to be proprietary, it has a major impact on data interpretation and comparability and therefore should be included as detailed as possible in future studies. Highest possible sample-frequency is recommended in multiples of 30 [35].

The younger the subjects, the shorter the epoch length to capture sporadic movements [35]. In light of the WHO recommendation asking for bouts of $\geq 10 \mathrm{~min}$ [37], sporadic movements become more negligible, and longer sampling epochs are suitable in adults [35]. All studies in ACHD and young adults reported to adhere to this employing 30-60 s sampling epochs. In pediatric studies epoch length differs (Table 3 ), and future studies should focus on using the shortest sampling epoch possible. It is recommended to follow the same epoch length and intensity classification criteria which were used in the original calibration study of the respective device. As internal algorithms differ by manufacturer, data processing is device specific. Therefore, it is advisable to use the default intensity classifications. Table 4 contains further recommendations for the application of objective PA assessment in clinical research.

\section{Are Patients with CHD Active Enough?}

With a few very specific exceptions, almost all patients with CHD can and should be encouraged by their physician and medical care-takers to achieve general population guidelines for PA $[3,38]$. Those are according to the World Health Organization as follows: children and adolescents aged 5-17 should accumulate at least $60 \mathrm{~min}$ of MVPA daily with greater benefits above $60 \mathrm{~min}$. Adults aged 18-64 should reach at least 150 min of moderateintensity aerobic PA throughout the week or do at least 
Table 3. PA assessment characteristics

\begin{tabular}{|c|c|c|c|c|c|c|}
\hline Study & Device & $\begin{array}{l}\text { Placement } \\
\text { (waterproof) }\end{array}$ & Assessment length & Data inclusion criteria & $\begin{array}{l}\text { Sampling } \\
\text { frequency, } \\
\mathrm{Hz}\end{array}$ & $\begin{array}{l}\text { Sampling } \\
\text { epoch, s }\end{array}$ \\
\hline \multicolumn{7}{|l|}{ Pediatric $C H D(n=8)$} \\
\hline Kao et al. [18] & RT3 & Hip (no) & $\begin{array}{l}3 \text { days starting on Friday waking } \\
\text { hours }\end{array}$ & - & - & - \\
\hline Ewalt et al. [19] & ActiGraph 7164 & Hip (no) & 7 consecutive days waking hours & $\begin{array}{l}\geq 10 \text { h of data for } 3 \text { weekdays and } 1 \\
\text { weekend day }\end{array}$ & - & 30 \\
\hline $\begin{array}{l}\text { Longmuir et al. } \\
{[20]}\end{array}$ & $\begin{array}{l}\text { Respironics actical } \\
2.1\end{array}$ & Hip (yes) & $\begin{array}{l}5 \text { school days and } 2 \text { nonschool days } \\
\text { waking hours }\end{array}$ & $\begin{array}{l}\geq 8 \text { h of data on } 3 \text { school days and } 1 \\
\text { nonschool day }\end{array}$ & - & 15 \\
\hline Stone et al. [21] & ActiGraph GT1M & Hip (no) & 7 consecutive days waking hours & $\begin{array}{l}\geq 5 \text { h on } \geq 4 \text { days incl. } 1 \text { weekend } \\
\text { day }\end{array}$ & - & 3 \\
\hline Klausen et al. [22] & ActiGraph 77 & Hip (no) & $\begin{array}{l}6 \text { a.m. - } 10 \text { p.m. for } 4 \text { weekdays and } \\
2 \text { weekend days at baseline and FU }\end{array}$ & $\begin{array}{l}\geq 10 \text { h of data on } \geq 1 \text { weekend day } \\
\text { and } 1 \text { weekday }\end{array}$ & - & 5 \\
\hline Banks et al. [23] & $\begin{array}{l}\text { Respironics actical } \\
2.1\end{array}$ & Hip (yes) & $\begin{array}{l}5 \text { school days and } 2 \text { nonschool days } \\
\text { waking hours }\end{array}$ & $\begin{array}{l}\geq 10 \text { h of data on } \geq 3 \text { school days } \\
\text { and } 1 \text { nonschool day }\end{array}$ & - & 15 \\
\hline Voss et al. [24] & $\begin{array}{l}\text { ActiGraph GT3X+ } \\
\text { and GT9X }\end{array}$ & Hip (no) & 7 consecutive days waking hours & $\geq 600 \mathrm{~min} /$ day of data on $\geq 3$ days & 30 & 15 \\
\hline Brudy et al. [25] & Garmin vivofit jr & Wrist (yes) & 7 consecutive days waking hours & 3 weekdays and 1 weekend day & - & 60 \\
\hline \multicolumn{7}{|l|}{$A C H D(n=5)$} \\
\hline Dua et al. [26] & ActiGraph 7164 & Hip (no) & 7 consecutive days waking hours & - & - & 60 \\
\hline Müller et al. [27] & RT3 & Hip (no) & 7 consecutive days waking hours & - & - & 60 \\
\hline Sandberg et al. [28] & ActiHeart & Chest (no) & $\begin{array}{l}4 \text { consecutive days } \\
24 \mathrm{~h}\end{array}$ & - & - & 30 \\
\hline $\begin{array}{l}\text { Opotowsky et al. } \\
{[29]}\end{array}$ & ActiGraph GT3X+ & Hip (no) & $\begin{array}{l}7 \text { consecutive days at baseline and } \\
\text { FU waking hours }\end{array}$ & $\geq 600 \mathrm{~min} /$ day of data on $\geq 3$ days & - & - \\
\hline Larsson et al. [30] & ActiHeart & Chest (no) & $\begin{array}{l}4 \text { consecutive days } \\
24 \mathrm{~h}\end{array}$ & - & - & 30 \\
\hline \multicolumn{7}{|c|}{ Pediatric and $A C H D(n=3)$} \\
\hline Müller et al. [31] & RT3 & Hip (no) & 3 consecutive days waking hours & - & - & 60 \\
\hline Duppen et al. [32] & ActiGraph GT3X & Hip (no) & 5 consecutive days waking hours & $\geq 8$ h/day on $\geq 3$ days & - & - \\
\hline Hedlund et al. [33] & ActiGraph GT3X & Wrist (no) & $\begin{array}{l}7 \text { consecutive days } \\
24 \mathrm{~h}\end{array}$ & - & 30 & 60 \\
\hline
\end{tabular}

PA, physical activity; $\mathrm{CHD}$, congenital heart disease; $\mathrm{ACHD}$, adults with congenital heart disease.

75 min of MVPA throughout the week or an equivalent combination of both intensities [37]. Further specific recommendations on indications, type, and intensity for PA and exercise in patients with CHD can be found in international guidelines $[4,39,40]$.

\section{Pediatric Patients with CHD}

None of the four CSS comparing pediatric patients with CHD to a RC found a difference between patients and healthy in daily PA or MVPA [18, 19, 21, 25]. Only one [25] found their patients reaching the WHO rec- ommendation for children and adolescents. The remaining three $[19,22,24]$ followed quite inactive cohorts in regards to their respective age-related PA recommendations. The cohort of Stone et al. [21] was also quite inactive but was compared to the Canadian PA Guidelines for the Early Years [41] with some of their patients being older than the used institutional recommendation.

Two CSS without RC evaluated PA in regard to CHD severity or CHD subgroups and did not find differences in mean MVPA [23, 24], while others [25] reported com- 
Table 4. Recommendations for the application of PA assessment in clinical research

\begin{tabular}{ll}
\hline Sampling protocol & $\begin{array}{l}\text { 7-day sampling period; } \geq 4 \text { valid days including 1 weekend day capturing all waking hours; clear } \\
\text { data inclusion criteria defined }\end{array}$ \\
\hline Device placement & $\begin{array}{l}\text { Possible trade-off between hip (better for intensity classification accuracy) \& wrist-worn (better } \\
\text { for wear-time compliance) devices to consider; ideally both in research settings }\end{array}$ \\
\hline Sampling frequency & Highest possible in multiples of 30 \\
\hline Epoch length & $\begin{array}{l}\text { Shorter (3-10 s) in children \& adolescents to capture sporadic movement; longer (30-60 s) in } \\
\text { adults }\end{array}$ \\
\hline Intensity classification \& data filters & Follow initial calibration study guidelines or default settings of the device \\
\hline PA guidelines/reference & $\begin{array}{l}\text { Age \& region/country-appropriate guidelines must be employed; preferably WHO criteria and } \\
\text { country specific guidelines; own recruited healthy reference cohort is advisable }\end{array}$ \\
\hline Confounders to consider & Age, sex, BMI, CHD severity, weather, and seasonality \\
\hline PA, physical activity. &
\end{tabular}

plex CHD to be associated with not meeting the WHO recommendation on PA.

While two studies $[24,25]$ report a decrease in daily PA with increasing age, Longmuir et al. [20] were able to keep MVPA constant through an intervention study over 22 months of long-term FU contrasting the expected decrease in daily PA with age. Furthermore, all three studies dealing with pediatric and ACHD (but generally rather young cohorts) found an age-related decrease in PA [3133].

Even though the influence of weather and seasonality on PA behavior has been demonstrated [42], only a few studies controlled their results $[20,24,25]$ therefor. One [20] found an association between spring season and PA in pediatric patients with CHD.

\section{Pediatric and ACHD}

Müller and co-workers [31] found $72 \%$ of patients with total cavopulmonary connection to reach $\geq 60 \mathrm{~min}$ activity $\geq 3$ MET on $\geq 5$ days per week [43]. Similarly, Duppen et al. [32] had $70 \%$ of patients meeting $\geq 60$ MVPA min/day at baseline before heading into intervention. Young male patients were significantly more active than female which was also confirmed in ACHD [28]. However, Duppen et al. [32] did not improve daily PA or percentage of MVPA time through 12 weeks of aerobic training in an already active young cohort. In contrast, Hedlund et al. [33] showed $90 \%$ of their young population to reach $\leq 60 \mathrm{MVPA} \mathrm{min} /$ day, an overall similar measured MVPA for patients and age-and-sex-matched RC, and an age-related MVPA decrease. Furthermore, patients spent less time sedentary and more time in light activity than RC [33].

\section{ACHD}

One of the three CSS [27] reported 76\% of ACHD to be fairly active reaching $\geq 30 \mathrm{MVPA} \mathrm{min} /$ day, while the other two [28, 30] found no differences in MVPA against a healthy RC and in the proportion that reached the WHO guideline [37]. The latter two also found about half of all ACHD and RC not reaching daily PA recommendations. It is important to note that both Sandberg et al. [28] and Larsson et al.[30] calculate the WHO recommendation as 21.4 MVPA min/day. However, the WHO recommends to split the $\geq 30 \mathrm{MVPA} \min /$ day in bouts of $\geq 10 \mathrm{~min}$ [37]. Therefore, these results need to be interpreted carefully.

The clinical controlled trial [26] and the RCT [29] conducted in ACHD did not use a healthy RC. Dua and colleagues [26] increased the adherence to the UK National Guidelines for PA [44] from 14 to 56\% and mean MVPA minutes almost doubled after 10 weeks intervention. Furthermore, patients with higher NYHA class were less active at baseline and more able to improve their PA through training. Contrarily, Opotowsky and colleagues [29] found only a trend toward more MVPA time, and no difference regarding PA at baseline and FU after 12 week cardiac rehabilitation intervention versus standard of care.

Generally, it seems like the older the cohorts, the less active CHD patients are both against healthy RC and in- 
stitutional PA recommendations. Few of the herein mentioned interventional studies tried to improve PA showing mixed results on the efficacy of these interventions. Large studies with a focus on CHD subgroups currently do not exist.

\section{$P A$ and Exercise Capacity}

Eight studies determined parameters for physical capacity, mostly through cardiopulmonary exercise testing in CHD patients, but four reported cardiopulmonary exercise testing outcomes separately $[20,22,29,32]$, while one [26] only examined maximal walking time without associating PA. Müller and colleagues [27] found a moderate relationship between daily activity and exercise capacity and PA to be reduced in patients with lower $\mathrm{VO}_{2 \text { peak }}[31]$, while Banks et al. [23] found only a trend in the association between MVPA and higher percent-predicted $\mathrm{VO}_{2}$. All 3 studies did not have a healthy RC.

Considering the importance of exercise capacity in predicting mortality in CHD patients [45], future studies should explore the association to PA in this population more in detail. In a similar physiological context, there is no information on the relationship between daily PA and cardiovascular risk in CHD patients. Exploring this triumvirate of objectively measured PA, exercise capacity, and cardiovascular risk holds potential for further studies and eventually improving medical aftercare in CHD patients.

\section{$P A$ and Quality of Life}

Müller et al. [27] found only a poor correlation between daily PA and several QoL domains in ACHD. In pediatric patients with $\mathrm{CHD}$, Banks and co-workers [23] showed higher MVPA to be associated with higher selfefficacy in pediatric patients with $\mathrm{CHD}$, while Müller and colleagues [31] found QoL to be related to daily activity in children with CHD younger than 14 years. Duppen et al. [32] confirmed weak correlation between self-estimated QoL and daily PA in adolescents and young ACHD. Hedlund and co-workers [33] sampled but did not analyze PA in relation to QoL. Therefore, clear answer on the relationship between objective PA and QoL in CHD patients can currently not be given leaving room for future, large scale studies.

\section{Limitations}

Sample size of the majority of included studies was generally small. Comparison between studies presented difficulties because device types and characteristics, data classification criteria, and the guidelines PA was measured against differed substantially.

The majority of studies were cross-sectional, five were interventional. The main goal of this review was to compare the status quo by evaluating quality of methods, outcome measures, and results and not to assess interventions of the separate studies. Therefore, we decided to critically appraise all articles according to the NHLBI Quality Assessment Tool for Observational Cohort and Cross-Sectional Studies. We acknowledge that to evaluate the intervention quality the NHLBI Quality Assessment of Controlled Intervention Studies is the appropriate tool for RCT or clinical controlled trial. However, both questionnaires contain similar key methodological items.

\section{Conclusion and Clinical Recommendation}

The methodology and assessment of PA was fair at best in most of the studies. Due to methodological differences of the studies, no clear answer on how active CHD patients really are can currently be given.

Objective PA measures are crucial in identifying patients at risk for inactivity. Therefore, larger studies adhering to consistent measuring and reporting standards exploring PA in CHD patients, also in the context of different angles such as exercise capacity or QoL, are needed. These studies should employ their own healthy RC and compare PA against institutional guidelines. Future studies need to consider device placement, sampling protocol and frequency, epoch length, intensity classification thresholds, valid-day inclusion criteria, and wear-time definition more carefully.

\section{Conflict of Interest Statement}

No conflict of interest. No competing interests for any of the authors.

\section{Funding Sources}

The authors L.B. and M.M. are funded by an unrestricted grant from the "Stiftung KinderHerz Deutschland gGmbH" and "Fördergemeinschaft Deutsche Kinderherzzentren e.V." These sponsors had no role in the study design, collection, analysis and interpretation of the data, in writing of the report, or the decision to submit the paper for publication. 


\section{Author Contributions}

J.M. was responsible for conception of the review and further monitored the search process and quality assessment of applicable studies. L.B. and M.M. screened and assessed the search results. L.B. also developed the search strategy and drafted the manuscript.
L.G.C. was the third reviewer to dissolve in case of disagreement between the first and the second reviewer and further gave valuable feedback for improving the manuscript. R.O., A.H., and P.E. gave important input for revising and improving the quality of the manuscript. All authors have read and approved the final version of the manuscript.

\section{References}

1 Forouzanfar MH, Forouzanfar MH, Alexander L, Anderson HR, Bachman VF, Biryukov $S$, et al. Global, regional, and national comparative risk assessment of 79 behavioural, environmental and occupational, and metabolic risks or clusters of risks in 188 countries, 1990-2013: a systematic analysis for the Global Burden of Disease Study 2013. Lancet. 2015;386(10010):2287-323.

2 Kohl HW 3rd, Craig CL, Lambert EV, Inoue S, Alkandari JR, Leetongin G, et al. The pandemic of physical inactivity: global action for public health. Lancet. 2012;380(9838):294305.

3 Takken T, Giardini A, Reybrouck T, Gewillig M, Hövels-Gürich HH, Longmuir PE, et al. Recommendations for physical activity, recreation sport, and exercise training in paediatric patients with congenital heart disease: a report from the Exercise, Basic \& Translational Research Section of the European Association of Cardiovascular Prevention and Rehabilitation, the European Congenital Heart and Lung Exercise Group, and the Association for European Paediatric Cardiology. Eur J Prev Cardiol. 2012;19(5):1034-65.

4 Longmuir PE, Brothers JA, de Ferranti SD, Hayman LL, Van Hare GF, Matherne GP, et al. Promotion of physical activity for children and adults with congenital heart disease: a scientific statement from the American Heart Association. Circulation. 2013;127(21):214759.

5 Henriksen A, Haugen Mikalsen M, Woldaregay AZ, Muzny M, Hartvigsen G, Hopstock LA, et al. Using fitness trackers and smartwatches to measure physical activity in research: analysis of consumer wrist-worn wearables. J Med Internet Res. 2018;20(3): e110.

6 Nascimento-Ferreira MV, De Moraes ACF, Toazza Oliveira PV, Rendo-Urteaga T, Gracia-Marco L, Forjaz CLM, et al. Assessment of physical activity intensity and duration in the paediatric population: evidence to support an a priori hypothesis and sample size in the agreement between subjective and objective methods. Obes Rev. 2018;19(6):810-24.

7 Uman LS. Systematic reviews and meta-analyses. J Can Acad Child Adolesc Psychiatry. 2011;20(1):57-9.

8 Moher D, et al. Preferred reporting items for systematic reviews and meta-analyses: the PRISMA statement. PLoS Med. 2009;6(7): e1000097.

Objective Physical Activity Assessment in Clinical CHD Research
9 Fan JX, Brown BB, Hanson H, KowaleskiJones L, Smith KR, Zick CD. Moderate to vigorous physical activity and weight outcomes: does every minute count? Am J Health Promot. 2013;28(1):41-9.

10 National heart, L., and Blood Institute. Quality assessment Tool for observational Cohort and cross-sectional studies. 2014.

11 Klausen SH, Mikkelsen UR, Hirth A, Wetterslev J, Kjærgaard H, Søndergaard L, et al. Design and rationale for the PREVAIL study: effect of e-Health individually tailored encouragements to physical exercise on aerobic fitness among adolescents with congenital heart disease: a randomized clinical trial. Am Heart J. 2012;163(4):549-56.

12 Takken T, Stephens S, Balemans A, Tremblay MS, Esliger DW, Schneiderman J, et al. Validation of the actiheart activity monitor for measurement of activity energy expenditure in children and adolescents with chronic disease. Eur J Clin Nutr. 2010;64(12):1494-500.

13 Voss C, Gardner RF, Dean PH, Harris KC. Validity of commercial activity trackers in children with congenital heart disease. Can J Cardiol. 2017;33(6):799-805.

14 Longmuir PE, Banks L, McCrindle BW. Cross-sectional study of motor development among children after the Fontan procedure. Cardiol Young. 2012;22(4):443-50.

15 Bay A, Sandberg C, Thilén U, Wadell K, Johansson B. Exercise self-efficacy in adults with congenital heart disease. Int J Cardiol Heart Vasc. 2018;18:7-11

16 Boyes NG, Stickland MK, Fusnik S, Hogeweide E, Fries JTJ, Haykowsky MJ, et al. Physical activity modulates arterial stiffness in children with congenital heart disease: a CHAMPS cohort study. Congenit Heart Dis. 2018;13(4):578-83.

17 Jacobsen RM, Ginde S, Mussatto K, Neubauer J, Earing M, Danduran M. Can a home-based cardiac physical activity program improve the physical function quality of life in children with Fontan circulation? Congenit Heart Dis. 2016;11(2):175-82.

18 Kao CC, Chang PC, Chiu CW, Wu LP, Tsai JC. Physical activity levels of school-age children with congenital heart disease in Taiwan. Appl Nurs Res. 2009;22(3):191-7.

19 Ewalt LA, Danduran MJ, Strath SJ, Moerchen V, Swartz AM. Objectively assessed physical activity and sedentary behaviour does not differ between children and adolescents with and without a congenital heart defect: a pilot examination. Cardiol Young. 2012;22(1):34-41.
20 Longmuir PE, Tyrrell PN, Corey M, Faulkner G, Russell JL, McCrindle BW. Home-based rehabilitation enhances daily physical activity and motor skill in children who have undergone the Fontan procedure. Pediatr Cardiol. 2013;34(5):1130-51.

21 Stone N, Obeid J, Dillenburg R, Milenkovic J, MacDonald MJ, Timmons BW. Objectively measured physical activity levels of young children with congenital heart disease. Cardiol Young. 2015;25(3):520-5.

22 Klausen SH, Andersen LL, Søndergaard L, Jakobsen JC, Zoffmann V, Dideriksen K, et al. Effects of eHealth physical activity encouragement in adolescents with complex congenital heart disease: the PReVaiL randomized clinical trial. Int J Cardiol. 2016;221: 1100-6.

23 Banks L, Rosenthal S, Manlhiot C, Fan CS, McKillop A, Longmuir PE, et al. Exercise capacity and self-efficacy are associated with moderate-to-vigorous intensity physical activity in children with congenital heart disease. Pediatr Cardiol. 2017;38(6):1206-14.

24 Voss C, Duncombe SL, Dean PH, de Souza AM, Harris KC. Physical activity and sedentary behavior in children with congenital heart disease. J Am Heart Assoc. 2017 Mar 6; 6(3): 0004665

25 Brudy L, Hock J, Häcker AL, Meyer M, Oberhoffer R, Hager A, et al. Children with congenital heart disease are active but need to keep moving: a cross-sectional study using wrist-worn physical activity trackers. J Pediatr. 2020;217:13-9.

26 Dua JS, Cooper AR, Fox KR, Graham Stuart A. Exercise training in adults with congenital heart disease: feasibility and benefits. Int J Cardiol. 2010;138(2): 196-205.

27 Müller J, Hess J, Hager A. Daily physical activity in adults with congenital heart disease is positively correlated with exercise capacity but not with quality of life. Clin Res Cardiol. 2012;101(1):55-61.

28 Sandberg C, Pomeroy J, Thilén U, Gradmark A, Wadell K, Johansson B. Habitual physical activity in adults with congenital heart disease compared with age- and sex-matched controls. Can J Cardiol. 2016;32(4):547-53.

29 Opotowsky AR, Rhodes J, Landzberg MJ, Bhatt AB, Shafer KM, Yeh DD, et al. A randomized trial comparing cardiac rehabilitation to standard of care for adults with congenital heart disease. World J Pediatr Congenit Heart Surg. 2018;9(2):185-93. 
30 Larsson L, Johansson B, Wadell K, Thilén U, Sandberg C. Adults with congenital heart disease overestimate their physical activity level. Int J Cardiol Heart Vasc. 2019;22:137.

31 Müller J, Christov F, Schreiber C, Hess J, Hager A. Exercise capacity, quality of life, and daily activity in the long-term follow-up of patients with univentricular heart and total cavopulmonary connection. Eur Heart J. 2009; 30(23):2915-20.

32 Duppen N, Etnel JR, Spaans L, Takken T, van den Berg-Emons RJ, Boersma E, et al. Does exercise training improve cardiopulmonary fitness and daily physical activity in children and young adults with corrected tetralogy of Fallot or Fontan circulation? A randomized controlled trial. Am Heart J. 2015;170(3): 606-14.

33 Hedlund ER, Lundell B, Villard L, Sjöberg G. Reduced physical exercise and health-related quality of life after Fontan palliation. Acta Paediatr. 2016;105(11):1322-8.

34 James WP, Ferro-Luzzi A, Waterlow JC. Definition of chronic energy deficiency in adults. Report of a working party of the International Dietary Energy Consultative Group. Eur J Clin Nutr. 1988;42(12):969-81.

35 Migueles JH, Cadenas-Sanchez C, Ekelund U, Delisle Nyström C, Mora-Gonzalez J, Löf M, et al. Accelerometer data collection and processing criteria to assess physical activity and other outcomes: a systematic review and practical considerations. Sports Med. 2017; 47(9): 1821-45.

36 Trost SG, Pate RR, Freedson PS, Sallis JF, Taylor WC. Using objective physical activity measures with youth: how many days of monitoring are needed? Med Sci Sports Exerc. 2000;32(2):426-31.

37 WHO guidelines approved by the guidelines review committee. In: Global recommendations on physical activity for health. Geneva: World Health Organization Copyright (c) World Health Organization; 2010.

38 Morrison ML, Sands AJ, McCusker CG, McKeown PP, McMahon M, Gordon J, et al. Exercise training improves activity in adolescents with congenital heart disease. Heart. 2013;99(15):1122-8.

39 Hirth A, Reybrouck T, Bjarnason-Wehrens B, Lawrenz W, Hoffmann A. Recommendations for participation in competitive and leisure sports in patients with congenital heart disease: a consensus document. Eur J Cardiovasc Prev Rehabil. 2006;13(3):293-9.

40 Budts W, Pieles GE, Roos-Hesselink JW, Sanz de la Garza M, D’Ascenzi F, Giannakoulas G, et al. Recommendations for participation in competitive sport in adolescent and adult athletes with Congenital Heart Disease (CHD): position statement of the Sports Cardiology \& Exercise Section of the European Association of Preventive Cardiology (EAPC), the Euro- pean Society of Cardiology (ESC) Working Group on Adult Congenital Heart Disease and the Sports Cardiology, Physical Activity and Prevention Working Group of the Association for European Paediatric and Congenital Cardiology (AEPC). Eur Heart J. 2020 Aug 26:ehaa501.

41 Tremblay MS, Leblanc AG, Carson V, Choquette L, Connor Gorber S, Dillman C, et al. Canadian physical activity guidelines for the early years (aged 0-4 years). Appl Physiol Nutr Metab. 2012;37(2):345-69.

42 Tucker P, Gilliland J. The effect of season and weather on physical activity: a systematic review. Public Health. 2007;121(12):909-22.

43 Pate RR, Freedson PS, Sallis JF, Taylor WC, Sirard J, Trost SG, et al. Compliance with physical activity guidelines: prevalence in a population of children and youth. Ann Epidemiol. 2002;12(5):303-8.

44 Department of Health. Physical activity, health improvement, and prevention. At least five a week. Evidence on the impact of physical activity and its relationship to health. A report from the Chief Medical Officer. Department of Health; 2004

45 Diller GP, Dimopoulos K, Okonko D, Li W, Babu-Narayan SV, Broberg CS, et al. Exercise intolerance in adult congenital heart disease: comparative severity, correlates, and prognostic implication. Circulation. 2005;112(6): $828-35$. 